\title{
Expression of STAT3, MMP-1 and TIMP-1 in gastric cancer and correlation with pathological features
}

\author{
QIN-WEI CAI，JUN LI，XUE-QIN LI，JIAN-QIANG WANG and YUAN HUANG
}

\begin{abstract}
Department of Gastroenterology, The Second Affiliated Hospital to Nanchang University, Nanchang 330006, P.R. China
\end{abstract}
Received December 9, 2011; Accepted March 19, 2012

DOI: $10.3892 / \mathrm{mmr} .2012 .849$

\begin{abstract}
The aim of this study was to investigate the mRNA and protein expression of STAT3, MMP-1 and TIMP-1 in gastric cancer (GC), and to explore the correlations between these proteins and the biological behaviors of GC. Reverse transcription-polymerase chain reaction was employed to detect the mRNA expression of STAT3, MMP-1 and TIMP-1 in GC tissues ( $n=30)$, adjacent normal tissues $(n=30)$ and superficial gastritis $(\mathrm{SG})$ tissues $(\mathrm{n}=30)$. Immunohistochemistry was performed using the SP method to measure the protein expression of STAT3 (unphosphorylated), MMP-1 and TIMP-1. The correlation between the pathological features of $\mathrm{GC}$ and STAT3, MMP-1 as well as TIMP-1, were evaluated. The mRNA expression of STAT3 in GC tissues $(0.821 \pm 0.128)$ was significantly higher compared to that in adjacent normal tissues $(0.355 \pm 0.100)$ and $\mathrm{SG}$ tissues $(0.398 \pm 0.096)(\mathrm{P}<0.05)$. The mRNA expression of MMP-1 in GC tissues $(0.749 \pm 0.133)$ was significantly increased compared to adjacent normal tissues $(0.335 \pm 0.106)$ and $S G$ tissues $(0.345 \pm 0.063)(\mathrm{P}<0.05)$. The mRNA expression of TIMP-1 in GC tissues $(0.386 \pm 0.125)$ was comparable to that in adjacent normal tissues $(0.343 \pm 0.078)$ and SG tissues $(0.345 \pm 0.061)$, but the mRNA expression of TIMP-1 in GC tissues was significantly correlated with the differentiation of GC cells and lymph node metastasis. STAT3, MMP-1 and TIMP-1 were significantly associated with the differentiation of GC cells and lymph node metastasis, but not related to age, gender and tumor size. The positive rate of unphosphorylated STAT3 expression was dramatically higher in GC tissues $(86.7 \%)$ compared to that in adjacent normal tissues $(16.7 \%)$ and SG tissues $(10.0 \%)(\mathrm{P}<0.05)$. The positive rate of MMP-1 protein expression in GC tissues $(63.3 \%)$ was significantly higher compared to that in adjacent normal tissues $(13.3 \%)$ and SG tissues $(16.7 \%)(\mathrm{P}<0.05)$. However, no significant difference was observed in the TIMP-1-positive
\end{abstract}

Correspondence to: Dr Yuan Huang, Department of Gastroenterology, The Second Affiliated Hospital to Nanchang University, Nanchang 330006, P.R. China

E-mail: dochuang123@yeah.net

Key words: gastric cancer, signal transducer and activator of transcription 3, matrix metalloproteinase-1, tissue inhibitor of metalloproteinase-1 rate among the three groups $(23.3,16.7$ and $10.0 \%$, respectively; P>0.05). STAT3 and MMP-1 may be involved in the development and metastasis of GC, and treatment targeting TIMP-1 may be a promising strategy.

\section{Introduction}

Gastric cancer (GC) is the most common malignancy in the gastrointestinal tract. It is the second leading cause of cancer-related death worldwide (1). China has a relatively high morbidity of GC (2); the mortality rate of GC is 25.21 per 100,000 individuals annually and is the leading cause of cancer-related death in China (3). With the wide application of electronic gastroscopy, an increasing number of GC patients acquire the chance for surgery. However, when compared to early GC, patients with advanced GC have a significantly reduced survival rate after surgery due to the susceptibility of $\mathrm{GC}$ to recurrence and metastasis.

Signal transducer and activator of transcription 3 (STAT3) is a member of the STAT family which consists of 7 members (STAT-1, -2, -3, -4, -5A, -5B and -6). It has been confirmed that the STAT family plays important roles in many biological processes, including cell growth, differentiation, survival and development (4). Evidence indicates the aberrant activation of STAT3 in GC and GC cell lines. STAT3 promotes the proliferation and anti-apoptosis of $\mathrm{GC}$ cells via regulating the expression of Bcl-2, Bcl-xL, survivin and cyclin D1, which then promotes lymph node metastasis and affects the prognosis of GC (5-8). STAT3 may also facilitate the expression of matrix metalloproteinase (MMP) family members, including MMP-2 (9), MMP-7 (10) and MMP-9 (6), which have crucial roles in the invasion and metastasis of GC. There is evidence indicating that the expression of STAT3 and MMP-1 is increased in colon cancer, which exerts a promotive effect on the invasion and metastasis of GC $(11,12)$. Studies have also demonstrated increased MMP-1 levels in peripheral blood in GC patients and elevated MMP-1 expression in GC tissues (13-15). However, the interaction between STAT3 and MMP-1 is seldom reported in GC.

MMP is a group of proteins that have the activities of proteolytic enzymes and degrade the basement membrane and extracellular matrix. MMP is regulated by its endogenous inhibitor - tissue inhibitors of metalloproteinase (TIMP). To date, a total of 4 TIMPs have been identified (TIMP-1, -2, -3 and -4$)$ (5). The functions of the 4 TIMPs on MMPs are 
Table I. Annealing temperature and primers for RT-PCR.

\begin{tabular}{|c|c|c|c|}
\hline Gene & Primers 5'-3' & Annealing temperature $\left({ }^{\circ} \mathrm{C}\right)$ & Anticipated size (bp) \\
\hline \multirow{2}{*}{ STAT3 } & TGGGCATATGCGGCCAGCAA & & \\
\hline & ACAAAAGCCCCGCCAGCTCA & 58 & 550 \\
\hline \multirow{2}{*}{ MMP-1 } & ACATCGTGTTGCGGCTCATGA & & \\
\hline & TTTGGGGTTTGTGGGCCGATGG & 58 & 192 \\
\hline \multirow[t]{2}{*}{ TIMP-1 } & GTTGTTGCTGTGGCTGATGATAG & & \\
\hline & TGTGGGACCTGTGGAAGTA & 56 & 266 \\
\hline \multirow[t]{2}{*}{$\beta$-actin } & GTGGACATCCGCAAAGAC & & \\
\hline & AAAGGGTGTAACGCAACTAA & 56 & 302 \\
\hline
\end{tabular}

overlapped (16), and TIMP-1 exerts a suppressive effect on MMP-1 activity (17).

In the present study, reverse transcription-polymerase chain reaction (RT-PCR) and immunohistochemistry were performed to determine the mRNA and protein expression of STAT3, MMP-1 and TIMP-1, respectively, in GC. The clinical significance of these proteins in GC may provide evidence for the pathogenesis of $\mathrm{GC}$, molecular-targeted therapy and evaluation of contributing factors of GC prognosis.

\section{Materials and methods}

Patients. A total of 30 patients with GC were recruited from May 2011 to August 2011. Fresh GC tissues $(n=30)$ were collected during surgery, including moderately differentiated adenocarcinoma $(n=13)$ and poorly differentiated adenocarcinoma $(n=17)$. At the same time, adjacent normal tissues were also obtained $5 \mathrm{~cm}$ away from the tumor $(\mathrm{n}=30)$. The mean age of the GC patients was $58.4 \pm 10.6$ years (range $39-78$ ) and there were 19 males and 11 females. All patients did not receive chemotherapy and radiotherapy before surgery and GC was pathologically confirmed. In addition, 30 patients with pathologically proven superficial gastritis (SG) who underwent gastroscopy were also recruited as controls. The mean age of the SG patients was $42.4 \pm 5.7$ years (range $32-52$ ) and there were 18 males and 12 females. Each tissue was divided into two: one was stored in liquid nitrogen for RT-PCR and the other was fixed in formalin and embedded in paraffin for immunohistochemistry.

Reagents. TRIzol, total RNA extraction kit, reverse transcription kit (Invitrogen), 2X TaqPCR MasterMix (TianGen), DNA Marker I (100-700 bp), rabbit anti-human Stat3 (unphosphorylated) monoclonal antibody, rabbit anti-human TIMP-1 polyclonal antibody, mouse anti-human MMP-1 monoclonal antibody (Santa Cruz), immunohistchemistry SP kit (Maxim) and DAB substrate kit were used in the present study.

Semi-quantitative RT-PCR. Total RNA was extracted from GC tissues, adjacent normal tissues and SG tissues according to the manufacturer's instructions and then identified by electrophoresis. RNA was subjected to reverse transcription into cDNA according to the manufacturer's instructions. RT-PCR was performed using the following procedures: $1 \mu \mathrm{l}$ of cDNA was mixed with $1 \mu \mathrm{l}$ of each primer, $12.5 \mu \mathrm{l}$ of $2 \mathrm{X}$
TaqPCR MasterMix and $9.5 \mu \mathrm{l}$ of RNase-free water (final volume $25 \mu \mathrm{l}$ ). The PCR conditions and primers are shown in Table I. Electrophoresis was performed according to the following procedures: $5 \mu \mathrm{l}$ of PCR products was subjected to $1 \%$ agarose gel electrophoresis and the bands were observed under a uviol lamp and then the images were captured. The optical density of the bands was analyzed using Bandleader 3.0 software. Expression of the target gene was normalized by that of $\beta$-actin as an internal reference representing the relative expression of the target gene (semi-quantitative analysis).

Immunohistochemistry. Immunohistochemistry was carried out using the streptavidin-perosidase (SP) method. In brief, tissues were embedded in paraffin and sectioned. The sections were then deparaffinized and hydrated. Antigen retrieval was carried out in citric acid buffer at high temperature. The remaining procedures were performed according to the manufacturer's instructions. The concentration of Stat3 (unphosphorylated), MMP-1 and TIMP-1 as primary antibody was 1:200. In the negative control group, the primary antibody was replaced with PBS. Cells positive for Stat3 (unphosphorylated), MMP-1 or TIMP-1 had yellow or brownish yellow granules in the cytoplasm. Fields at a high magnification (x200) were selected and the proportion of positive cells was calculated: negative, $<10 \%$; positive, $\geq 10 \%$.

Statistical analysis. Data are expressed as the means \pm standard deviation, and statistical analysis was carried out with SPSS version 13.0 software. Differences in mRNA expression were compared by t-test between two groups and differences in the proportion of positive cells were compared by the Chi-square test. A value of $\mathrm{P}<0.05$ was considered to indicate statistical significance.

\section{Results}

mRNA expression of STAT3, MMP-1 and TIMP-1. The positive rate of mRNA expression of STAT3, MMP-1 and TIMP-1 was $100 \%$ in GC tissues, adjacent normal tissues and SG tissues. The mRNA expression of STAT3 in GC tissues $(0.821 \pm 0.128)$ was markedly higher than that in adjacent normal tissues $(0.355 \pm 0.100)(\mathrm{P}<0.05)$ and SG tissues $(0.398 \pm 0.096)(\mathrm{P}<0.05)$, but the mRNA expression of STAT3 was comparable between adjacent normal tissues and SG tissues $(\mathrm{P}>0.05)$. The mRNA expression of MMP-1 in GC tissues $(0.749 \pm 0.133)$ was 


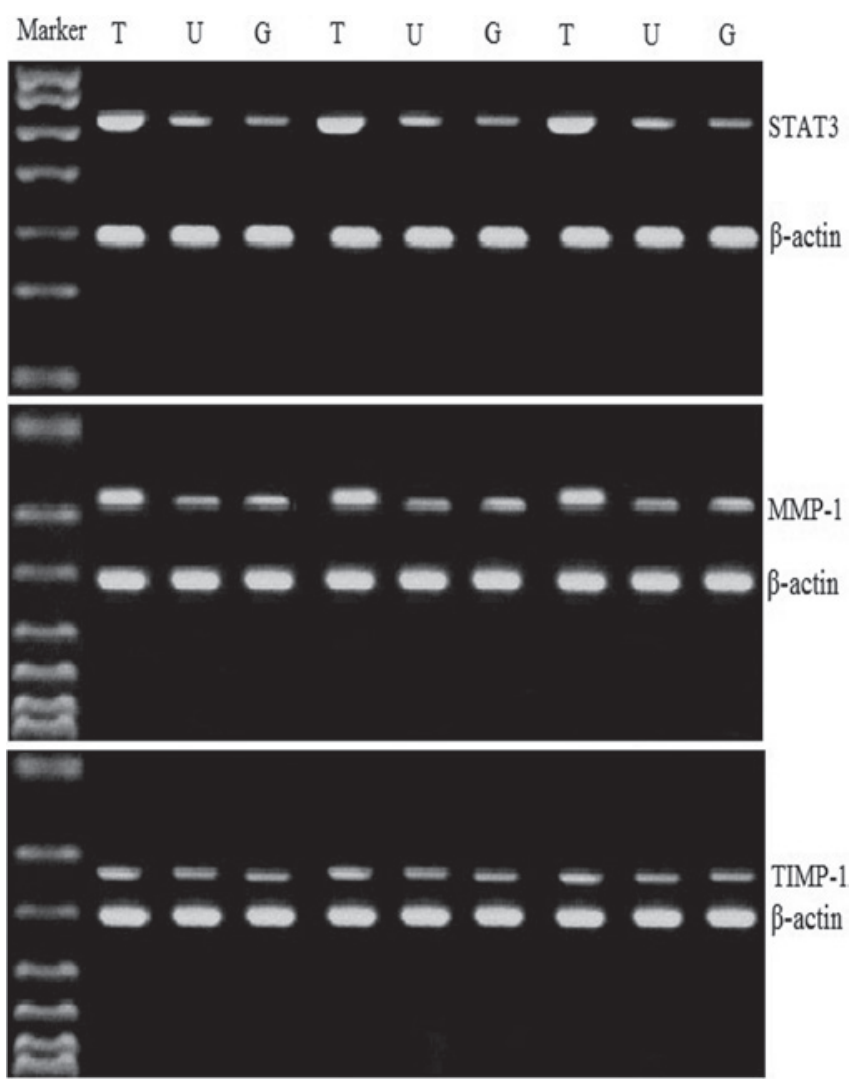

Figure 1. mRNA expression of STAT3, MMP-1 and TIMP-1 in different tissues (T, GC tissues; U, adjacent normal tissues; G, SG tissues). GC, gastric cancer; SG, superficial gastritis.

significantly increased when compared to adjacent normal tissues $(0.335 \pm 0.106)(\mathrm{P}<0.05)$ and $\mathrm{SG}$ tissues $(0.345 \pm 0.063)$ $(\mathrm{P}<0.05)$, but there was no significant difference between adjacent normal tissues and SG tissues $(\mathrm{P}>0.05)$. The mRNA expression of TIMP-1 in GC tissues $(0.386 \pm 0.125)$ was similar to that in adjacent normal tissues $(0.343 \pm 0.078)$ and SG tissues $(0.345 \pm 0.061)(\mathrm{P}>0.05)$. However, the mRNA expression of
TIMP-1 was related to lymph node metastasis and differentiation of GC cells in GC tissues (Fig. 1, Tables II and III).

Protein expression of STAT3, MMP-1 and TIMP-1. The proportion of STAT3-positive cells in GC tissues, adjacent normal tissues and SG tissues was $86.7 \%$ (26/30), $16.7 \%(5 / 30)$ and $10.0 \%(3 / 30)$, respectively. The positive rate in GC was significantly higher than that in adjacent normal tissues and SG tissues $(\mathrm{P}<0.05)$. The proportion of MMP-1-positive cells in GC tissues was $63.3 \%$ (19/30), which was markedly higher than that in adjacent normal tissues $13.3 \%$ (4/30) and SG tissues $16.7 \%(5 / 30)(\mathrm{P}<0.05)$. The proportion of TIMP-1-positive cells in GC tissues, adjacent normal tissues and SG tissues was $23.3 \%(7 / 30), 16.7 \%(5 / 30)$ and $10.0 \%$ (3/30), respectively, showing no significant difference $(\mathrm{P}>0.05)$ (Fig. 2).

Correlations between mRNA expression of STAT3, MMP-1 and TIMP-1 and pathological features of GC. The mRNA expression of STAT3, MMP-1 and TIMP-1 was closely related to the biological behaviors of GC, but was not associated with age, gender and tumor size. In addition, the mRNA expression of STAT3 and MMP-1 was associated with the differentiation and lymph node metastasis of GC cells. The mRNA expression of STAT3 and MMP-1 in poorly differentiated adenocarcinoma or GC with lymph node metastasis was significantly higher than that in moderately differentiated adenocarcinoma or GC without lymph node metastasis. $(\mathrm{P}<0.05)$. However, the mRNA expression of TIMP-1 in moderately differentiated GC or GC without lymph node metastasis was dramatically higher than that in poorly differentiated adenocarcinoma or GC with lymph node metastasis $(\mathrm{P}<0.05)$ (Table IV).

\section{Discussion}

Certain cytokines, growth factors and steroids bind to corresponding receptors on the cell membrane and then phosphorylate Janus kinase (JAK). The phosphorylated JAK subsequently phosphorylates the 705th amino acid at STAT3

Table II. mRNA expression of STAT3, MMP-1 and TIMP-1 in gastric cancer (GC) tissues, adjacent normal tissues and superficial gastritis (SG) tissues.

\begin{tabular}{lccc}
\hline Group & STAT3 & MMP-1 & TIMP-1 \\
\hline GC tissues & $0.821 \pm 0.128$ & $0.749 \pm 0.133$ & $0.386 \pm 0.125$ \\
Adjacent normal tissues & $0.355 \pm 0.100$ & $0.335 \pm 0.106$ & $0.343 \pm 0.078$ \\
SG tissues & $0.398 \pm 0.095$ & $0.345 \pm 0.063$ & $0.345 \pm 0.061$ \\
\hline
\end{tabular}

Table III. Protein expression of Stat3 (unphosphorylated), MMP-1 and TIMP-1 in gastric cancer (GC) tissues and adjacent normal tissues $(n=30)$.

\begin{tabular}{|c|c|c|c|c|c|c|c|c|c|c|c|c|}
\hline \multirow[t]{2}{*}{ Group } & \multicolumn{2}{|c|}{ STAT3 } & \multirow[t]{2}{*}{$\chi^{2}$-test } & \multirow[t]{2}{*}{ P-value } & \multicolumn{2}{|c|}{ MMP-1 } & \multirow[t]{2}{*}{$\chi^{2}$-test } & \multirow[t]{2}{*}{ P-value } & \multicolumn{2}{|c|}{ TIMP-1 } & \multirow[t]{2}{*}{$\chi^{2}$-test } & \multirow[t]{2}{*}{ P-value } \\
\hline & + & - & & & + & - & & & + & - & & \\
\hline GC tissues & 26 & 4 & 29.43 & 0.00 & 19 & 11 & 15.86 & 0.00 & 7 & 23 & 0.42 & 0.52 \\
\hline Adjacent tissues & 5 & 25 & & & 4 & 26 & & & 5 & 25 & & \\
\hline
\end{tabular}




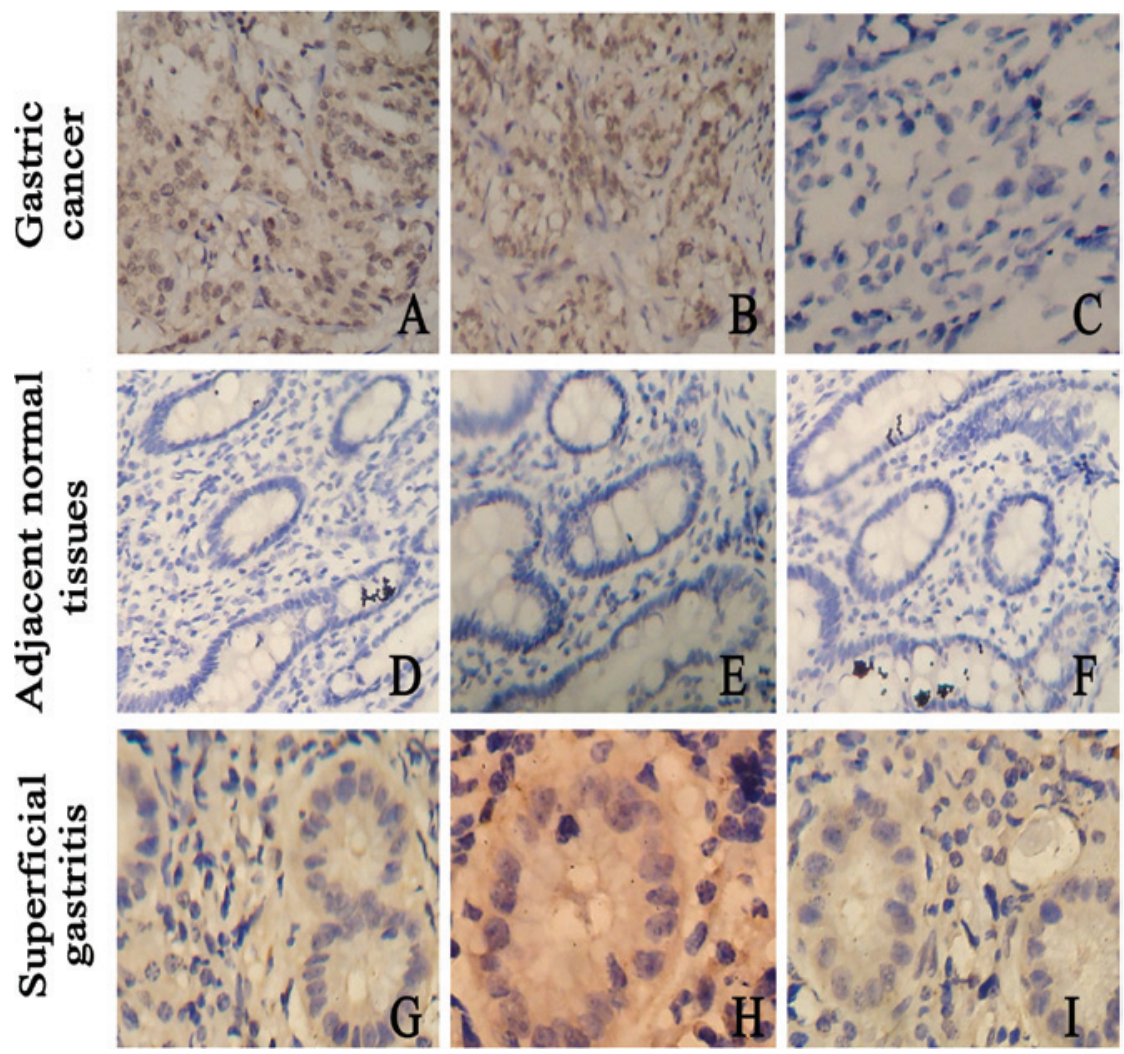

Figure 2. Protein expression of (A, D and G) Stat3 (unphosphorylated), (B, E and H) MMP-1 and (C, F and I) TIMP-1 in gastric cancer, adjacent normal and superficial gastritis tissues.

Table IV. Correlation between mRNA expression of STAT3, MMP-1 and TIMP-1 and pathological features of gastric cancer.

\begin{tabular}{|c|c|c|c|c|c|c|c|}
\hline $\begin{array}{l}\text { Pathological } \\
\text { features }\end{array}$ & no. & $\begin{array}{c}\text { STAT3 } \\
\text { expression }\end{array}$ & P-value & $\begin{array}{c}\text { MMP-1 } \\
\text { expression }\end{array}$ & P-value & $\begin{array}{c}\text { TIMP-1 } \\
\text { expression }\end{array}$ & P-value \\
\hline \multicolumn{8}{|l|}{ Gender } \\
\hline Male & 19 & $0.855 \pm 0.120$ & \multirow[t]{2}{*}{0.055} & $0.781 \pm 0.128$ & \multirow[t]{2}{*}{0.080} & $0.365 \pm 0.133$ & \multirow[t]{2}{*}{0.234} \\
\hline Female & 11 & $0.763 \pm 0.125$ & & $0.693 \pm 0.127$ & & $0.422 \pm 0.105$ & \\
\hline \multicolumn{8}{|l|}{ Age (years) } \\
\hline$<55$ & 10 & $0.804 \pm 0.156$ & \multirow[t]{2}{*}{0.611} & $0.758 \pm 0.131$ & \multirow[t]{2}{*}{0.803} & $0.368 \pm 0.057$ & \multirow[t]{2}{*}{0.479} \\
\hline$\geq 55$ & 20 & $0.830 \pm 0.115$ & & $0.745 \pm 0.137$ & & $0.395 \pm 0.148$ & \\
\hline \multicolumn{8}{|c|}{ Tumor size (cm) } \\
\hline$<5$ & 18 & $0.812 \pm 0.122$ & \multirow[t]{2}{*}{0.643} & $0.750 \pm 0.129$ & \multirow[t]{2}{*}{0.970} & $0.393 \pm 0.105$ & \multirow[t]{2}{*}{0.714} \\
\hline$\geq 5$ & 12 & $0.835 \pm 0.141$ & & $0.748 \pm 0.144$ & & $0.376 \pm 0.154$ & \\
\hline \multicolumn{8}{|c|}{ Differentiation } \\
\hline Moderate & 13 & $0.737 \pm 0.140$ & \multirow[t]{2}{*}{0.003} & $0.673 \pm 0.122$ & \multirow[t]{2}{*}{0.004} & $0.452 \pm 0.149$ & \multirow[t]{2}{*}{0.020} \\
\hline Poor & 17 & $0.886 \pm 0.069$ & & $0.807 \pm 0.112$ & & $0.336 \pm 0.073$ & \\
\hline \multicolumn{8}{|c|}{ Lymph node metastasis } \\
\hline No & 19 & $0.762 \pm 0.125$ & \multirow[t]{2}{*}{0.000} & $0.677 \pm 0.111$ & \multirow[t]{2}{*}{0.000} & $0.442 \pm 0.121$ & \multirow[t]{2}{*}{0.000} \\
\hline Yes & 11 & $0.924 \pm 0.032$ & & $0.874 \pm 0.044$ & & $0.289 \pm 0.047$ & \\
\hline
\end{tabular}

resulting in STAT3 activation [pSTAT3(Tyr705)]. The activated STAT3 forms dimers which then translocate into the nucleus and bind to the promoters of target genes involved in the transcription of target genes (4). In cancer cells, STAT3 is continuously activated leading to the increased expression of Bcl-2 (an anti-apoptosis protein), cyclin D1 (a cell cycle regulatory protein), survivin, vascular endothelial growth factor
(VEGF) and members of the MMP family. This may exert an anti-apoptotic effect, promote cell proliferation, regulate cell cycle, facilitate angiogenesis in cancer, and degrade the basement membrane and extracellular matrix, which play crucial roles in the occurrence and development of cancers $(6,18-20)$.

It was generally considered that pSTAT3 (Tyr705) is the activated form of STAT3. In recent years, several studies 
report that unphosphorylated-STAT3 (UP-STAT3) may also serve as a transcription factor to regulate the expression of target genes (21). There is evidence indicating that UP-STAT3 is highly expressed in several malignancies, including colon cancer (21) and chronic lymphocytic leukemia. This may be attributed to UP-STAT3 which forms dimers to enter the nucleus or bind to nuclear factor- $\kappa \mathrm{B}$ resulting in transcription activation $(19,22,23)$.

There are 24 members in the MMP family which have conservative gene structure enzyme activities. These members are involved in the degradation of the basement membrane and extracellular matrix. In normal cells, MMP is lowly expressed and TIMP can bind to MMP at a ratio of 1:1 to suppress MMP activity. The balance between MMP and TIMP is pivotal for the normal physiological functions of cells $(15,24)$. STAT3 regulates the transcription of MMP-1, which has been reported in colon cancer. STAT3 may directly act on MMP-1 or indirectly facilitate MMP-1 expression via activator protein (AP)-1 (a transcription factor) $(11,25)$.

Our results showed that the mRNA expression of STAT3 and MMP-1 in GC was markedly increased and also positively associated with the differentiation and lymph node metastasis of GC cells. However, the TIMP-1 expression was comparable among GC tissues, adjacent normal tissues and SG tissues ( $\mathrm{P}>0.05)$. Of note, TIMP-1 expression was negatively related to the differentiation and lymph node metastasis of GC cells. The protein expression of UP-STAT3 and MMP-1 in GC tissues was significantly increased when compared to adjacent normal tissues and SG tissues $(\mathrm{P}<0.05)$. This indicates that STAT3 and MMP-1 may be involved in the development and lymph node metastasis of GC, or the continuous high expression of STAT3 and MMP-1 may result in susceptibility to invasion and metastasis of GC. In GC, STAT3 is continuously activated leading to increased MMP-1 expression, but TIMP-1 expression remains unchanged. Thus, the balance between MMP-1 and TIMP-1 is disrupted and the degradation of the extracellular matrix by MMP-1 is enhanced, resulting in invasion and metastasis.

Taken together, in the present study we investigated the mRNA and protein expression of STAT3, MMP-1 and TIMP-1 in GC tissues, adjacent normal tissues and SG tissues, and also evaluated the correlations between these proteins and clinicopathological features of GC. We speculate that STAT3 and MMP-1 may be involved in the development and metastasis of poorly differentiated gastric adenocarcinoma affecting the prognosis of GC. TIMP-1 inhibits MMP-1 activity and the increased mRNA and protein expression of MMP-1 is related to the differentiation and lymph node metastasis of GC cells. Our findings provide evidence for the targeted therapy of TIMP-1 in GC.

\section{References}

1. Shah MA and Schwartz GK: Treatment of metastatic esophagus and gastric cancer. Semin Oncol 31: 574-587, 2004.

2. Huang S and JW Y: Situation and dispute of gastric cancer therapy between Oriental countries and Western countries. J Int Oncol 36: 689-692, 2009.
3. Lv W and L C: Current status and recent developments in molecular targeted therapy against gastric cancer. World Chin J Dig 15: 2672-2678, 2007.

4. Darnell JE Jr: STATs and gene regulation. Science 277: 1630-1635, 1997.

5. Deng JY, Sun D, Liu XY, Pan Y and Liang H: STAT-3 correlates with lymph node metastasis and cell survival in gastric cancer. World J Gastroenterol 16: 5380-5387, 2010.

6. Lee J, Kang WK, Park JO, et al: Expression of activated signal transducer and activator of transcription 3 predicts poor clinical outcome in gastric adenocarcinoma. APMIS 117: 598-606, 2009.

7. Kanda N, Seno H, Konda Y, et al: STAT3 is constitutively activated and supports cell survival in association with survivin expression in gastric cancer cells. Oncogene 23: 4921-4929, 2004.

8. Kim DY, Cha ST, Ahn DH, et al: STAT3 expression in gastric cancer indicates a poor prognosis. J Gastroenterol Hepatol 24: 646-651, 2009.

9. Huang W, Yu LF, Zhong J, et al: Stat3 is involved in angiotensin II-induced expression of MMP2 in gastric cancer cells. Dig Dis Sci 54: 2056-2062, 2009.

10. Shi M, Liu D, Duan H, et al: Catecholamine up-regulates MMP-7 expression by activating AP-1 and STAT3 in gastric cancer. Mol Cancer 9: 269, 2010

11. Zugowski C, Lieder F, Muller A, et al: STAT3 controls matrix metalloproteinase-1 expression in colon carcinoma cells by both direct and AP-1-mediated interaction with the MMP-1 promoter. Biol Chem 392: 449-459, 2011.

12. Tsareva SA, Moriggl R, Corvinus FM, et al: Signal transducer and activator of transcription 3 activation promotes invasive growth of colon carcinomas through matrix metalloproteinase induction. Neoplasia 9: 279-291, 2007.

13. Kemik O, Kemik AS, Sumer A, et al: Levels of matrix metalloproteinase-1 and tissue inhibitors of metalloproteinase-1 in gastric cancer. World J Gastroenterol 17: 2109-2112, 2011.

14. Zhou GQ and L W: Expression of MMP1 and TIMP1 genes in gastric cancer and its clinic significance. Chin J Hemorheol 19: 78-81, 2009.

15. Nagase $\mathrm{H}$ and Woessner JF Jr: Matrix metalloproteinases. J Biol Chem 274: 21491-21494, 1999.

16. Brew K, Dinakarpandian D and Nagase H: Tissue inhibitors of metalloproteinases: evolution, structure and function. Biochim Biophys Acta 1477: 267-283, 2000.

17. Bourboulia D and Stetler-Stevenson WG: Matrix metalloproteinases (MMPs) and tissue inhibitors of metalloproteinases (TIMPs): positive and negative regulators in tumor cell adhesion. Semin Cancer Biol 20: 161-168, 2010.

18. Takeda K and Akira S: STAT family of transcription factors in cytokine-mediated biological responses. Cytokine Growth Factor Rev 11: 199-207, 2000.

19. Lassmann S, Schuster I, Walch A, et al: STAT3 mRNA and protein expression in colorectal cancer: effects on STAT3inducible targets linked to cell survival and proliferation. J Clin Pathol 60: 173-179, 2007.

20. Zhang $\mathrm{C}$ and Zhong CH: STAT3: a critical transcription activator in angiogenesis. Med Res Rev 28: 185-200, 2008.

21. Yang J, Chatterjee-Kishore M, Staugaitis SM, et al: Novel roles of unphosphorylated STAT3 in oncogenesis and transcriptional regulation. Cancer Res 65: 939-947, 2005.

22. Liu Z, Hazan-Halevy I, Harris DM, et al: STAT-3 activates NF-kappaB in chronic lymphocytic leukemia cells. Mol Cancer Res 9: 507-515, 2011.

23. Braunstein J, Brutsaert S, Olson R and Schindler C: STATs dimerize in the absence of phosphorylation. J Biol Chem 278: 34133-34140, 2003.

24. Surlin V, Ioana M and Plesea IE: Genetic patterns of metalloproteinases and their tissular inhibitors - clinicopathologic and prognostic significance in colorectal cancer. Rom J Morphol Embryol 52: 231-236, 2011.

25. Tsareva SA, Wagner S, Muller A, Corvinus F and Friedrich K: Cell-cell contacts induce STAT3 activity in colon carcinoma cells through an autocrine stimulation loop. J Cancer Res Clin Oncol 137: 857-863, 2011. 